\title{
THE CONCEPTUAL MODEL OF AN APPLICATION TO INCREASE PUBLIC AWARENESS ON ANTIBIOTIC RESISTANCE
}

\author{
Ummul Hanan Mohamad", ${ }^{*}$, Aliimran Nordin ${ }^{2}$, Azlina Ahmad ${ }^{3}$ \\ 1 Institute of IR4.0, Universiti Kebangsaan Malaysia, Malaysia \\ Email: ummulhanan@ukm.edu.my \\ 2 Transforming Customer Journey, CIMB, Malaysia \\ Email: aliimran.nordin@cimb.com \\ 3 Institute of IR4.0, Universiti Kebangsaan Malaysia, Malaysia \\ Email: azlinaivi@ukm.edu.my \\ * Corresponding Author
}

\section{Article Info:}

Article history:

Received date: 19.08 .2020

Revised date: 13.09 .2020

Accepted date: 14.09 .2020

Published date: 15.09.2020

\section{To cite this document:}

Mohamad, U. H., Nordin, A., \& Ahmad, A. (2020). The Conceptual Model of An Application to Increase Public Awareness on Antibiotic Resistance Journal of Information System and Technology Management, 5 (18), 33-45.

DOI: $10.35631 / J I S T M .518004$.

\begin{abstract}
:
The emergence of antibiotics resistance has been one of the global health concerns. Hence, this paper analyzed the users' requirements to design a conceptual model of an application that can increase public awareness of the antibiotic resistance issue. The study involves 123 participants, in which they completed an online-based questionnaire to address the study's objectives. Two contributing factors associated with antibiotic resistance via common public malpractices are identified, including taking antibiotics for common viral diseases and not finishing antibiotics treatment. Hence, the conceptual model's design considers the need to educate users with correct information, engage users, and integrate elements that allow for voluntary behavior changes. For that reason, the gamification technique is incorporated into the design of the conceptual awareness model.
\end{abstract}

Keywords:

Android Application; Gamification; Mhealth; Public Awareness; Software Engineering

\section{Introduction}

Antibiotic has been a versatile treatment to overcome bacterial infections hitherto. However, it is observed that antibiotic resistance is escalating year by year (Donnelly, L., Davies, M., \& Wasley, 2017). More bacterial pathogens are becoming less sensitive to the antibiotic treatments, resulting in the emergence of "superbugs" or multi-drug resistant bacteria. Furthermore, O'Neill (2016) reported that nearly a million deaths occurred yearly due to 


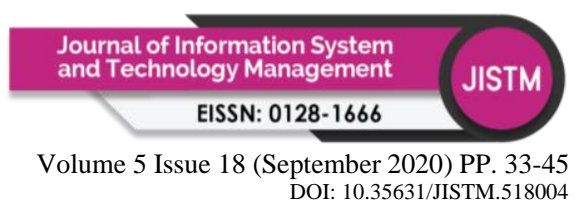

antibiotic resistance. Hence, it is predicted that this condition will eventually lead to 10 million deaths per annum by 2050 .

According to Islahudin et al. (2014), antibiotic resistance awareness remained low despite antibiotic resistance's alarming issue. Indirectly, this demonstrated a potential abuse in antibiotics usage (WHO, 2016) through the habits inflicted by the users (patients) or the prescribers (health practitioners). Studies by Lim \& The (2012) and Qamar et al. (2014) revealed several common habits that contributed to antibiotic resistance, such as stop taking antibiotics once feeling better, sharing of leftovers antibiotics among family members and the misuse of antibiotics to treat viral infections.

Despite many vigorous on-site campaigns conducted to increase antibiotic resistance (Bodendorf \& Kaiser, 2010), many failed to hold the users' interest. They were very sporadic and slow to show their impact. Hence, a better awareness model to increase public attention on the antibiotic resistance issue is necessary. In agreement with that, the mobile app is deemed a versatile tool in this digital era (Kao et al., 2018), especially for mHealth purposes (Abu Hassan Shaari et al., 2016). The mobile app can overcome many of the limitations faced by conventional awareness campaigns, such as rapidly sharing information (Man-Ching et al., 2018) and reaching wider demographic groups in a shorter period (Thiruvanackan \& Mohd. Yusof, 2017).

This study gathers the requirements based on the users' knowledge, practice and perspectives. It then analyses the findings to design a conceptual model of an application to increase public awareness of antibiotic resistance. The remaining sections of this paper are organized as follows. Section 2 describes the literature review of the existing antibiotic-related app available in the market and how gamification has been embedded for health awareness purposes. Meanwhile, Section 3 is devoted to the methodology. The findings are revealed in Section 4. Then, Section 5 includes the discussion. Section 6 provides the concluding remarks and highlights potential future work.

\section{Literature Review}

There is a need to identify the requirements based on user perspectives (Serral et al., 2010) to materialize a user-centered design. Many of the existing apps in the market often did not consider this as necessary before the development, thus resulting in low users' satisfaction and impede app usage (Peng et al., 2016).

Based on the search for antibiotics related app in the available app repositories, it was discovered that Google Play Store for Android contained the most number of related apps (51), Windows Mobile (11), Apple App Store (8) and Blackberry World (4). The majority of the apps were created to assist the health practitioners; functioned mainly as treatment guidelines and antibiotic dictionaries. The antibiotic-related app existed as dosing calculators and medication reminders (Zulkifli et al., 2018). Surprisingly, none of the available apps was developed to increase public awareness of the antibiotic resistance issue. Hence, this strengthens the need for this study.

A mobile app is a versatile tool to share information and reach wider demographic users rapidly. Thus, using a mobile app allows us to overcome those limitations faced by the conventional awareness program approaches. The findings through the requirement gathering will provide first-hand information that will help shape a conceptual framework for a mobile 


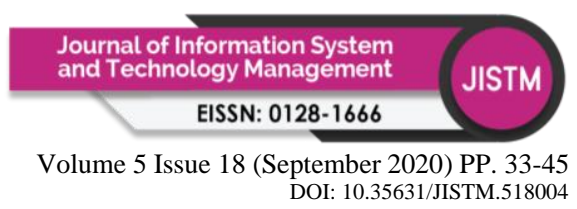

app. According to Tarute et al. (2017), user engagement to the mobile app is essential, mostly when intended for awareness purposes.

User engagement can be driven through gamification (S. Kim \& Baek, 2018). According to Deterding et al. (2011), gamification is defined as an activity that includes game elements in non-gaming contexts. Empirical evidence has revealed the positive effect of gamification on user motivation in various aspects such as education, rehabilitation (Korn \& Tietz, 2017), medical training, marketing and health awareness (Brauner et al., 2013). Notably, Gooch et al. (2016) asserted that gamification could ensure that every user can perform a gamified task and achieve objectives that give the inconstant value of motivation.

Undeniably, game-based activities or digital games can boost the healthcare industry by increasing patients' compliance with treatments, helping patients learn about their health conditions (Boratto et al., 2017), motivating patients, and engaging medical providers. A growing body of literature has suggested that gamification can educate patients, prevent illnesses, and control diseases. Johnson et al. (2016) agreed that the gamification approach promotes behavior change toward healthy wellbeing. The main idea of gamification is to enrich a non-gaming activity with motivational affordance. Thus, its design features are important for users to increase their engagement and motivation when performing the task.

One of the contributions gamification can make in the healthcare industry is to address obesity among children. This issue causes several health complications such as heart problems, Type 2 diabetes, hypertension, depression, fatty liver disease with a greater risk of psychological and social difficulties (Carrino et al., 2014). The researchers argued that suitable action should be taken to ensure that children are aware of the appropriate diet and physical activity to minimize their risk of obesity. To drive adolescents towards a healthy lifestyle through a reliable ICT system, Carrino et al. (2014) employed gamification as a design strategy to facilitate the dynamic of a game linked with physical activity that alters healthier behavioral patterns. Using the gamification approach, adolescents were more interested in engaging and forming physical activities.

Moreover, H. Kim et al. (2015) investigated the effectiveness and sustainability of an intervention to prevent obesity among children. They developed a smartphone application named "HAPPYME". This app employed gamification and focused on self-monitoring strategies relating to diet, physical activity, and socio-emotional support. The results showed that the detailed design of "HAPPYME" could facilitate smartphone application-supported child behavioral modification in obesity prevention and control.

Lee et al. (2017) examined how gamification affected users' intention to employ mobile healthcare applications (mHealth) and their impacts on their health, age, and gender. They reported that gamification factors in mHealth could intensify enjoyment, encouraging participation in health management. Moreover, gamification benefited healthy people in terms of wellbeing management. According to Lee et al. (2017) statistical evidence, healthy people enjoyed the gamified health activities and engagements with other people at the enjoyment of 0.725 compared to the unhealthy group at 0.400 .

The design of gamification must be carefully considered to allow us to obtain a positive outcome. Richards et al. (2014) suggested that designers should focus on gamification by identifying the system requirements, recognizing the organization's needs, understanding the target users, and addressing the problem.

Copyright (C) GLOBAL ACADEMIC EXCELLENCE (M) SDN BHD - All rights reserved 
Furthermore, Morschheuser et al. (2017) proposed the first stage of gamification is to understand the needs, motivations, behaviors, and characteristics of the users. Therefore, the first step is to follow an iterative process with high user involvement and early design ideas. A questionnaire survey is a possible tool to gather data from a substantial number of disparate users (Adams \& Cox, 2008). It helps achieve effectiveness, efficiency, and satisfaction. Since the survey allows collecting quantitative and qualitative data (Jones et al., 2013), the users' needs and requirements can be addressed accordingly. The findings from the requirement gathering can help set the app design's objectives and the priority for the users. These findings can also highlight exciting concepts and provide insights into specific issues such as antibiotic resistance awareness.

\section{Methodology}

The study was carried out according to Figure 1 in which three main steps were included; designing the requirement gathering questionnaire, performing requirement gathering and requirement analysis. The findings from these will help shape the conceptual model of antibiotic resistance awareness app.

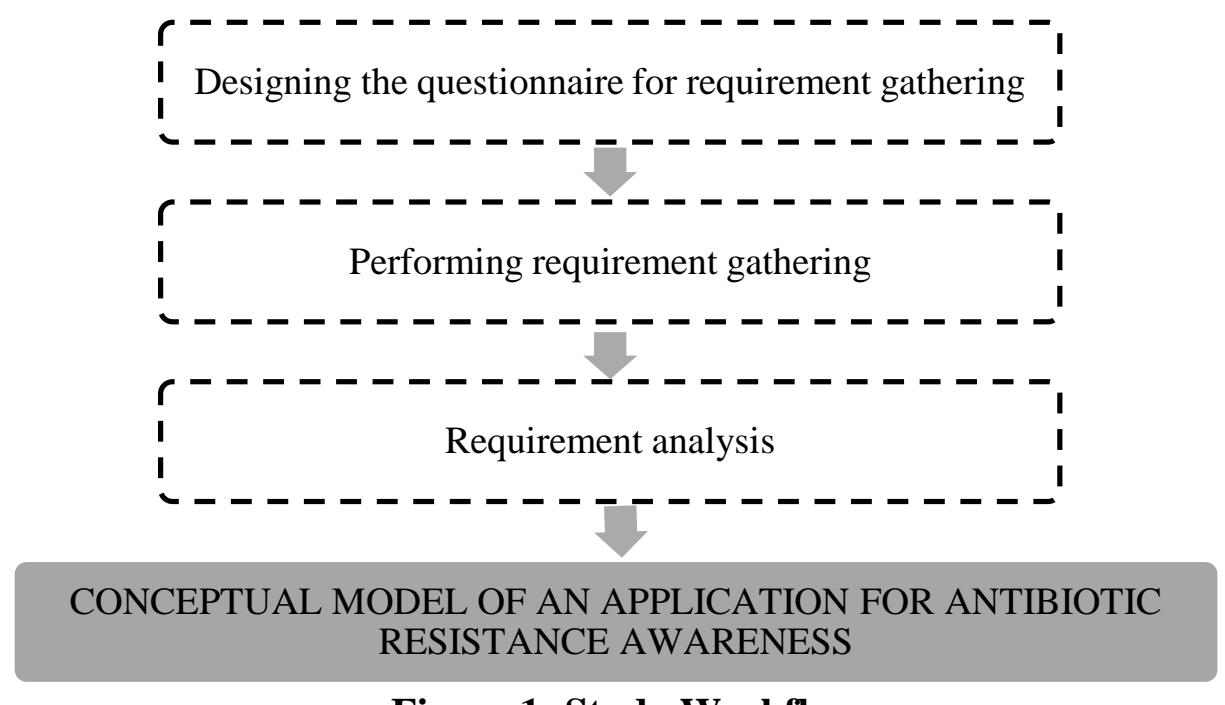

Figure 1: Study Workflow

\section{Designing The Questionnaire for Requirement Gathering}

The questionnaire was designed and adapted from several validated surveys that were previously used in several countries such as the US (WHO, 2015), China (Huang et al., 2013), Italy (Scaioli et al., 2015), India (Afzal Khan et al., 2013) and Malaysia (Lim \& Teh, 2012; Qamar et al., 2014). Since this study is performed among the Malaysian community, the questionnaire is tailored to suit the local population to ensure its applicability. It was designed in dual languages; English and Malay, the two common languages to Malaysians. The questionnaire comprises of four parts and contained both open-ended and close-ended questions. The preliminary part explained the questionnaire's purpose to the participants and informed them of the consent details.

The first part of the questionnaire recorded the participants' socio-demographic characteristics, such as age group, gender, education level, employment, and household income. Meanwhile, the second part consisted of six closed-ended questions to gather information about the users' knowledge of antibiotics and practice when using antibiotics. The questions include the frequency of taking antibiotics, the reason for taking antibiotics and whether the participants 


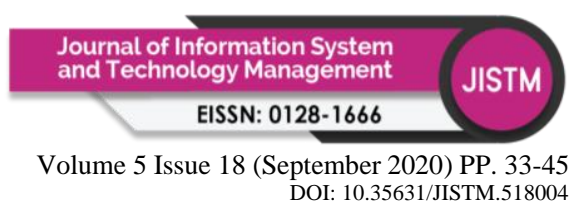

personally asked antibiotics from the doctor. There are three statements used to evaluate the participant's response in the five-point Likert scale ( $1=$ strongly disagree...5= strongly agree).

The third part studied the usage of the mobile application by the participants. The findings from this part helped us identify the users' needs and expectations in the mobile app. One open-ended question was included to gather the users' perspective on antibiotic resistance awareness mobile app. The full questionnaire can be obtained per request to the corresponding author.

\section{Performing Requirement Gathering}

The requirement gathering is performed through the online-based questionnaire. The invitation to the participants was carried out through email and social media postings such as LinkedIn, Facebook and WhatsApp. Before the questionnaire responses were collected, potential participants were first informed that their online survey involvement was voluntary. They were allowed to decline participation only by not clicking the consent button. At any point in the study, the participation withdrawal can be made without any personal consequences. If requested by the participants, their data can be destroyed immediately.

\section{Requirement Analysis}

The requirement analysis was conducted based on the three parts; demographic, antibiotic knowledge/practice and mobile app usage. Significant findings were highlighted to address the issue of being integrated into the gamified mobile app. The analysis will include identifying the problems that lead to antibiotic resistance and choosing the suitable gamification element for the design of the conceptual model.

\section{Results}

\section{Requirement Analysis of the Participants' Demographic}

The requirements gathering collected responses from 126 participants; however, three participants declined participation by refusing to provide their consent. Hence, this resulted in a total response rate of $97.6 \%$. The participants' demographic is shown in Figure 2. Out of 123 participants, more than $60 \%$ were female $(n=82)$. Participants' ethnicity was predominantly Malay (inclusive of Bumiputera Sabah and Sarawak) at nearly 88\%. The majority of the participants fell in the age group between 18-40 years old. More than $90 \%$ of participants had a minimum of at least a tertiary education, which denoted for a high education level. 


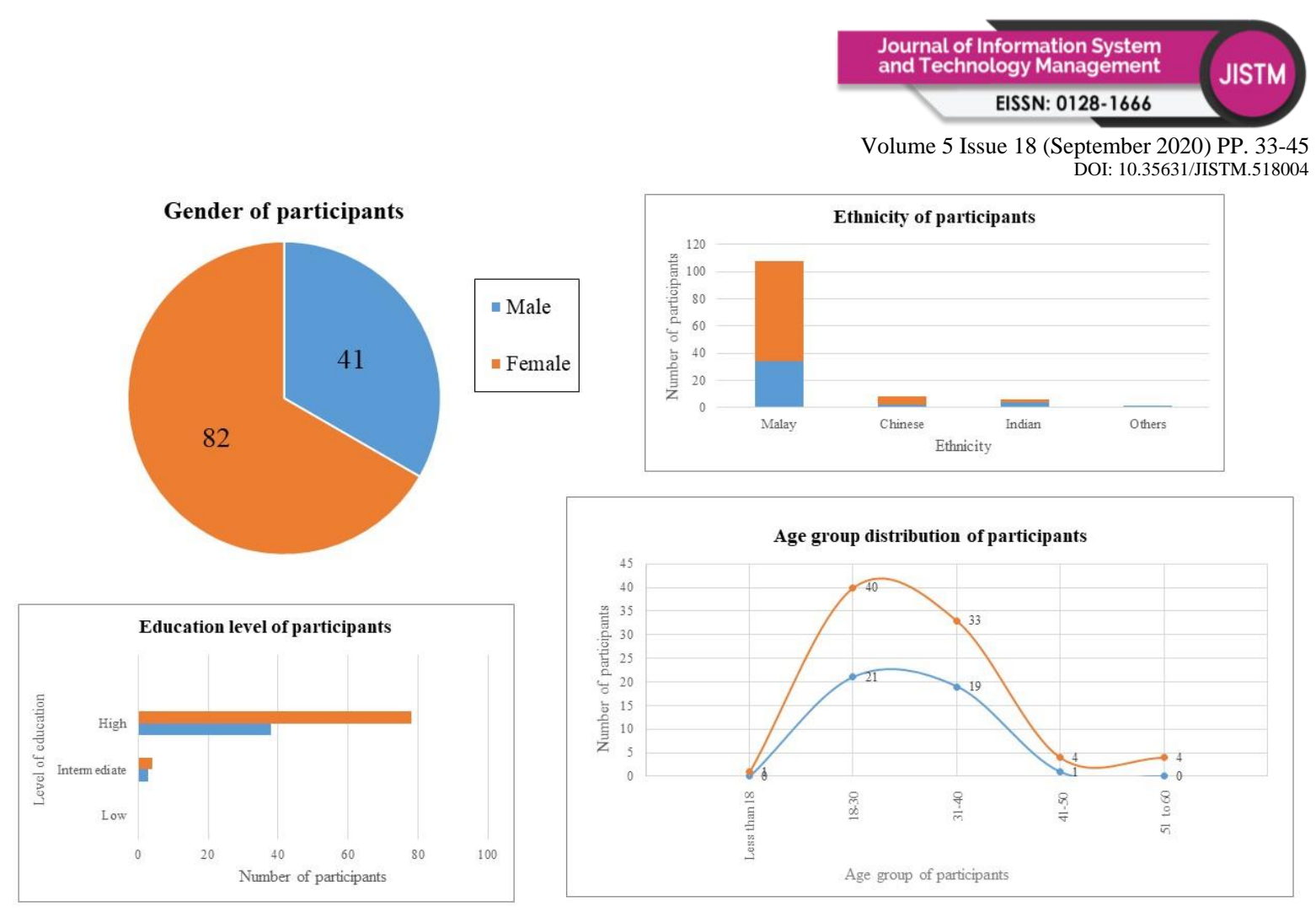

Figure 2: Visualization of Participants' Demographic

Requirement Analysis of the Participants' Knowledge and Practices Concerning Antibiotics The requirement gathering also considers the importance of identifying the participants' knowledge and practices concerning antibiotics and antibiotics resistance. As shown in Figure 3 , nearly $85 \%$ of participants had a general understanding of what is antibiotics. This finding is also supported by more than $85 \%$ disagreement rate on Statement 1 (Panadol/Uphamol is an example of an antibiotic). Panadol/Uphamol is a brand of paracetamol which is common among Malaysians. As paracetamol is a medicine generally prescribed by the doctor to relieve pain and fever, this typically signified that the participants had a basic idea of what is antibiotics and the general look of antibiotic medicine.

On the other hand, the participants' knowledge of antibiotics resistance was tested through Statement 3. More than half of the participants (61\%) viewed antibiotic resistance as a condition where the body no longer responds to the antibiotic treatment. Yet, antibiotic resistance is correctly defined as the condition in which the bacteria that are usually susceptible to a particular antibiotic treatment can now resist the antibiotic's action. 


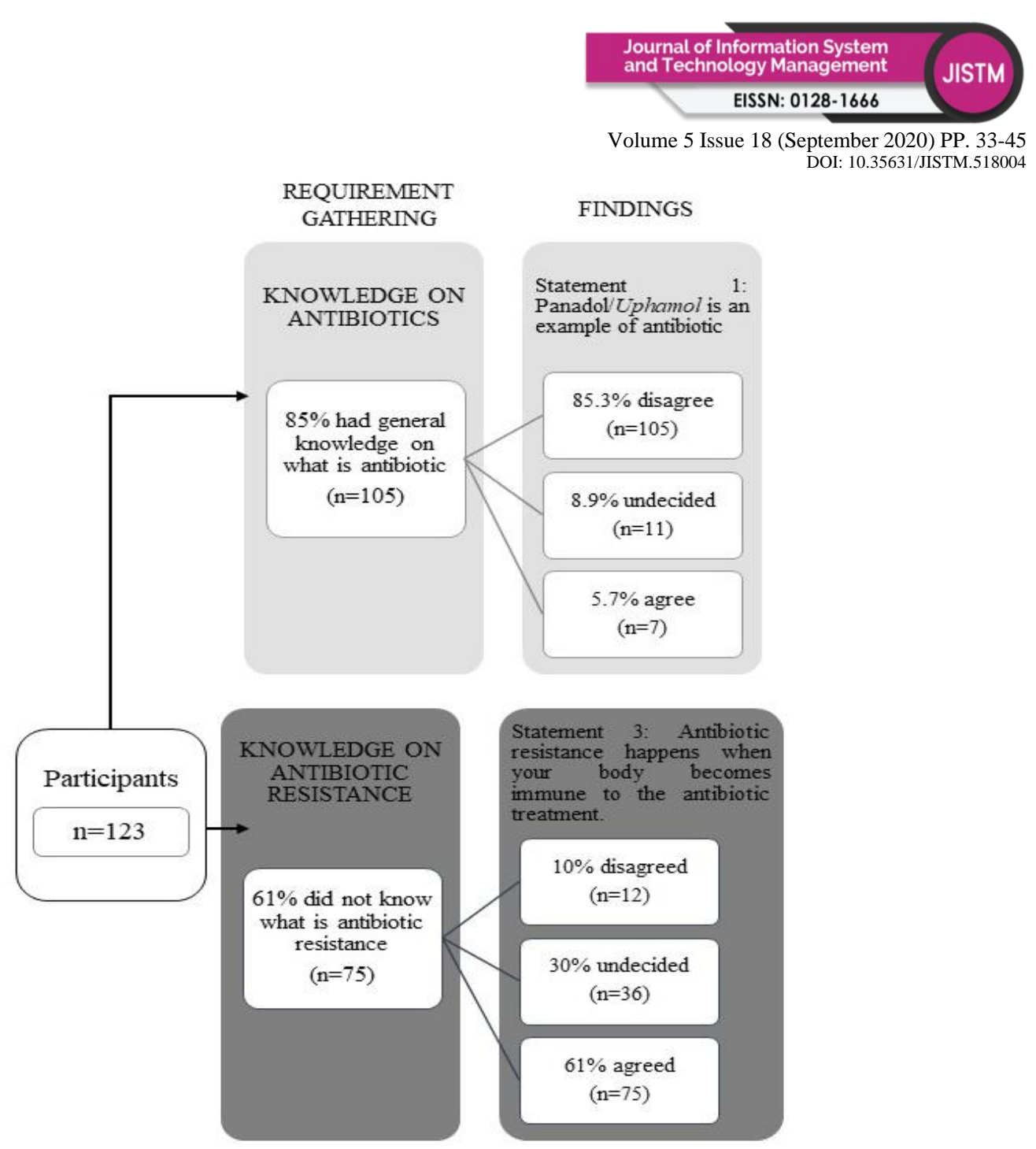

Figure 3: Visualization of Participants' Knowledge of Antibiotics

Besides, the requirement analysis also revealed how the participants used antibiotics. At least $76 \%$ of participants took antibiotics for common viral diseases such as cold, fever and sore throat. In agreement with that, the responses from Statement 2 (Antibiotics can speed up my recovery when I had cold, sore throat or fever) revealed a similar trend at $64 \%$ agreement. It was discovered that there are three categories of antibiotics users; Category 1 included those who took antibiotics when prescribed by the doctor (61\%), Category 2 was those who took antibiotics regardless of being prescribed or not by the doctor (35\%). Category 3 involved those who did not receive antibiotics $(4 \%)$. Focusing on Category 2, more than $60 \%$ of participants stopped taking antibiotics immediately once they felt better. This condition is alarming since this practice contributed hugely to the rise of antibiotic resistance.

Furthermore, it was also found that approximately $30 \%$ of participants personally asked antibiotics from their doctors. This situation happens due to a strong belief that the antibiotics aided their fast recovery at a $47 \%$ agreement rate. Again, this finding is also on par with the responses on Statement 2 (64\% agreement rate). 


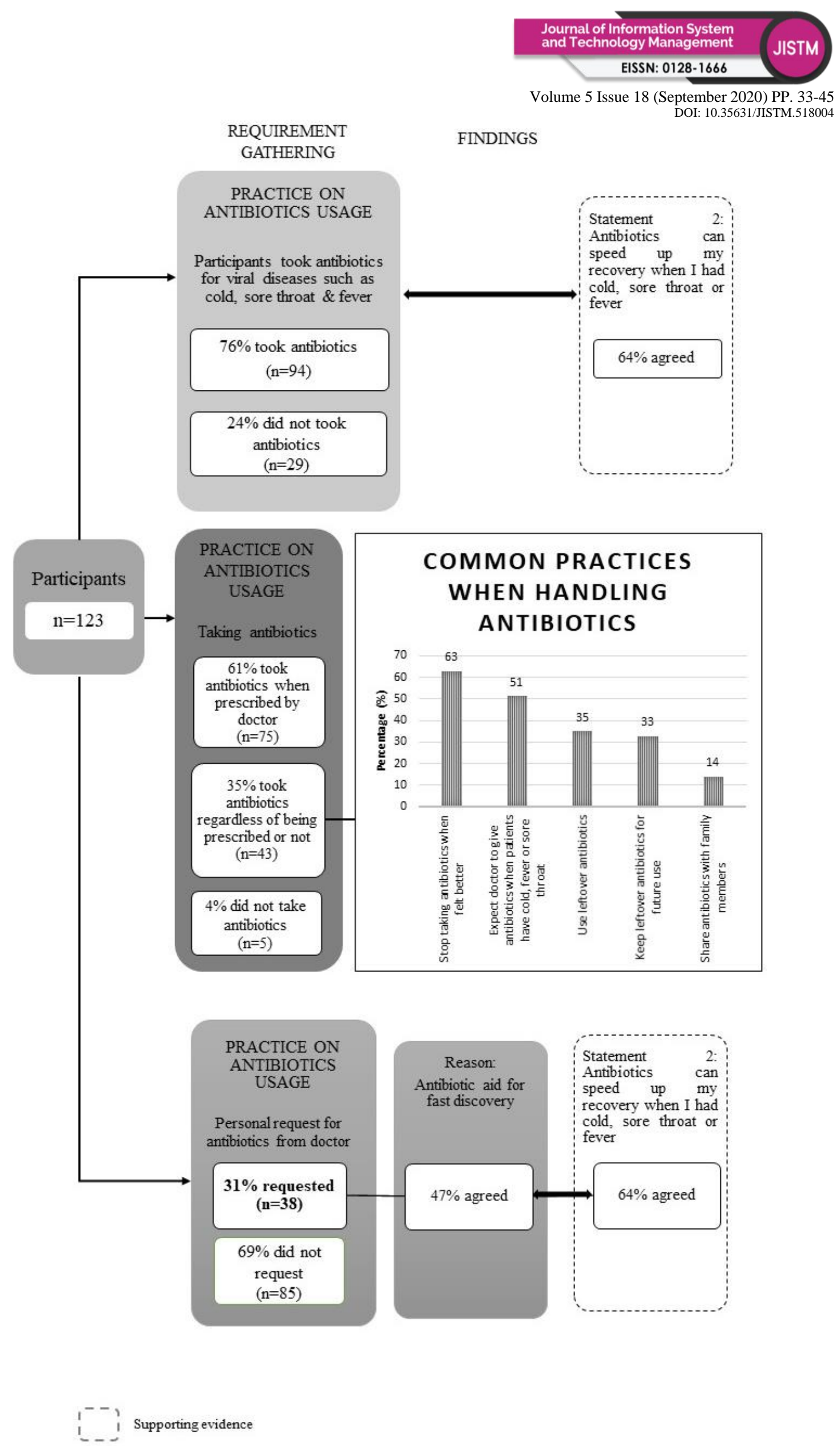

Figure 4: Visualization of Participants' Practices On Antibiotics Usage 


\section{Requirement Analysis On the Participants' Usage of Mobile Application}

Volume 5 Issue 18 (September 2020) PP. 33-45 DOI: $10.35631 /$ JISTM.518004

Requirement analysis on the participants' mobile application usage revealed that all participants owned smartphones and the majority were Android users $(58 \%, \mathrm{n}=71)$. The findings demonstrated that $70 \%$ of participants used at least one mobile app that relates to health in their smartphones on health-related apps usage. It indirectly displayed health concerns among the participants. Moreover, the responses also depicted the tendency to abandon an app according to this order; no longer need it, unattractive features, and app price. The app is taking too much space and difficult to use (see Figure 5). Therefore, it was crucial to ensure that the app design met the users' expectations (Tarute et al., 2017), considering that the target group for this antibiotic resistance awareness app was broad as it involves the public.

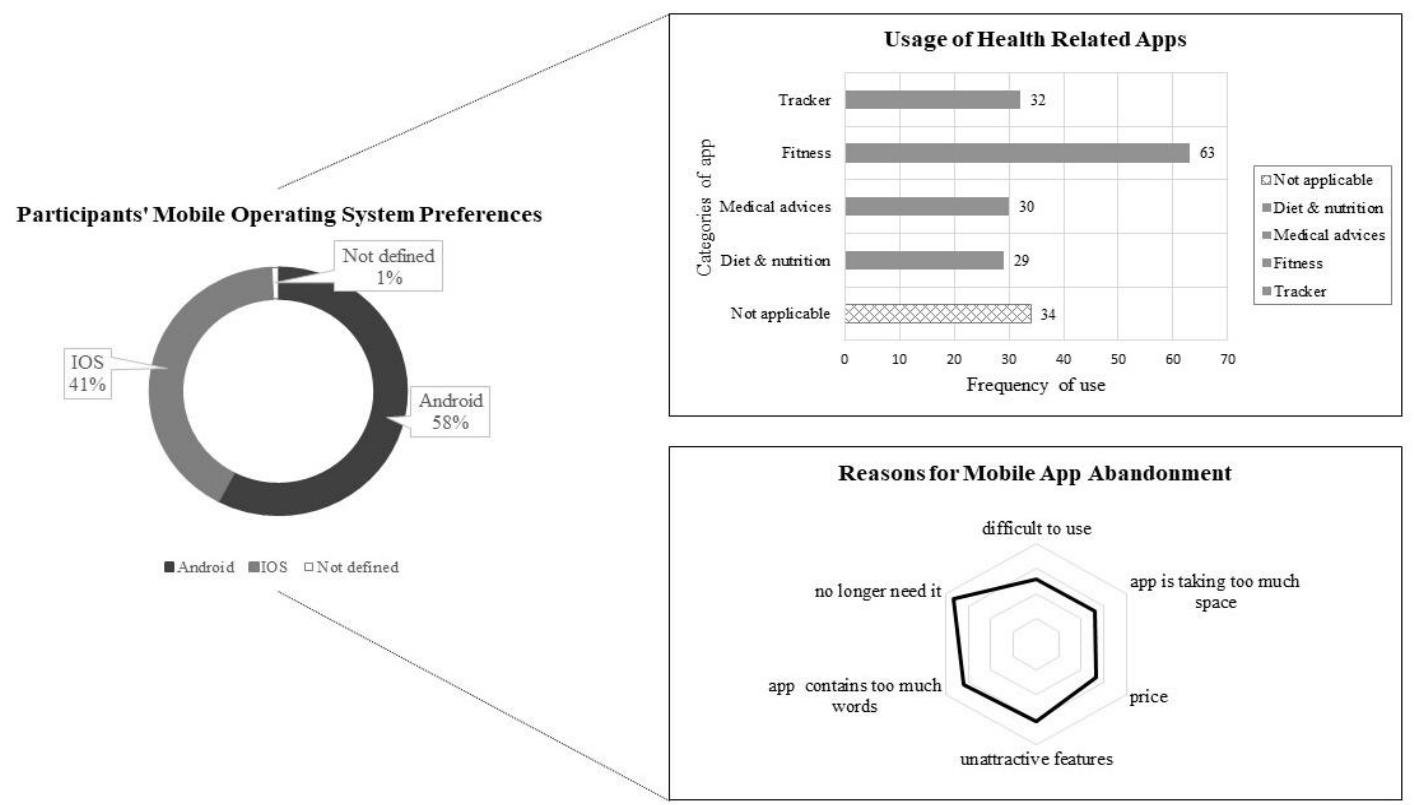

Figure 5: Visualization of Participants' Mobile Application Usage

To add, a summary from the open-ended questions detailed a few feedbacks for considerations in the design of the conceptual model for an application on antibiotic resistance awareness. Although many participants were suggesting for features such as the antibiotic tracker, dosing calculator as well as digital information on types of antibiotics and side effects of antibiotics, these types of features were made available in other existing antibiotic apps in the market. Nonetheless, some critical components that can be considered include the multilingual versions, information in laymen terms and motivational elements for proper antibiotic usage.

\section{Antibiotic Resistance Awareness Conceptual Model}

Figure 6 illustrated the conceptual model for the application of antibiotic resistance awareness called SEDAR. SEDAR stands for 'Stop and End the Drastic Antibiotics Resistances'. The requirement analysis findings determined the features for graphical user interfaces and the major components inside the application. The major components included the learning task, sharing task and motivation element. The learning contents on antibiotics and resistances are conveyed in the introductory narrative and learning modules.

Meanwhile, the gamification approach was chosen to act as the motivation element in this model. The primary aim is to encourage users to engage in learning and sharing tasks. For the learning task, the feedback provides a real-time response to convey the users' performance in learning. Meanwhile, for sharing task, the feedback depicted simple visualizations that 


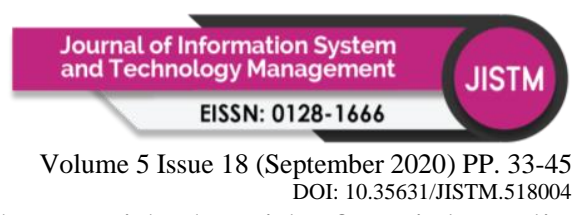

encourage the users' actions to share what they learn to others, with the aid of social media platforms.

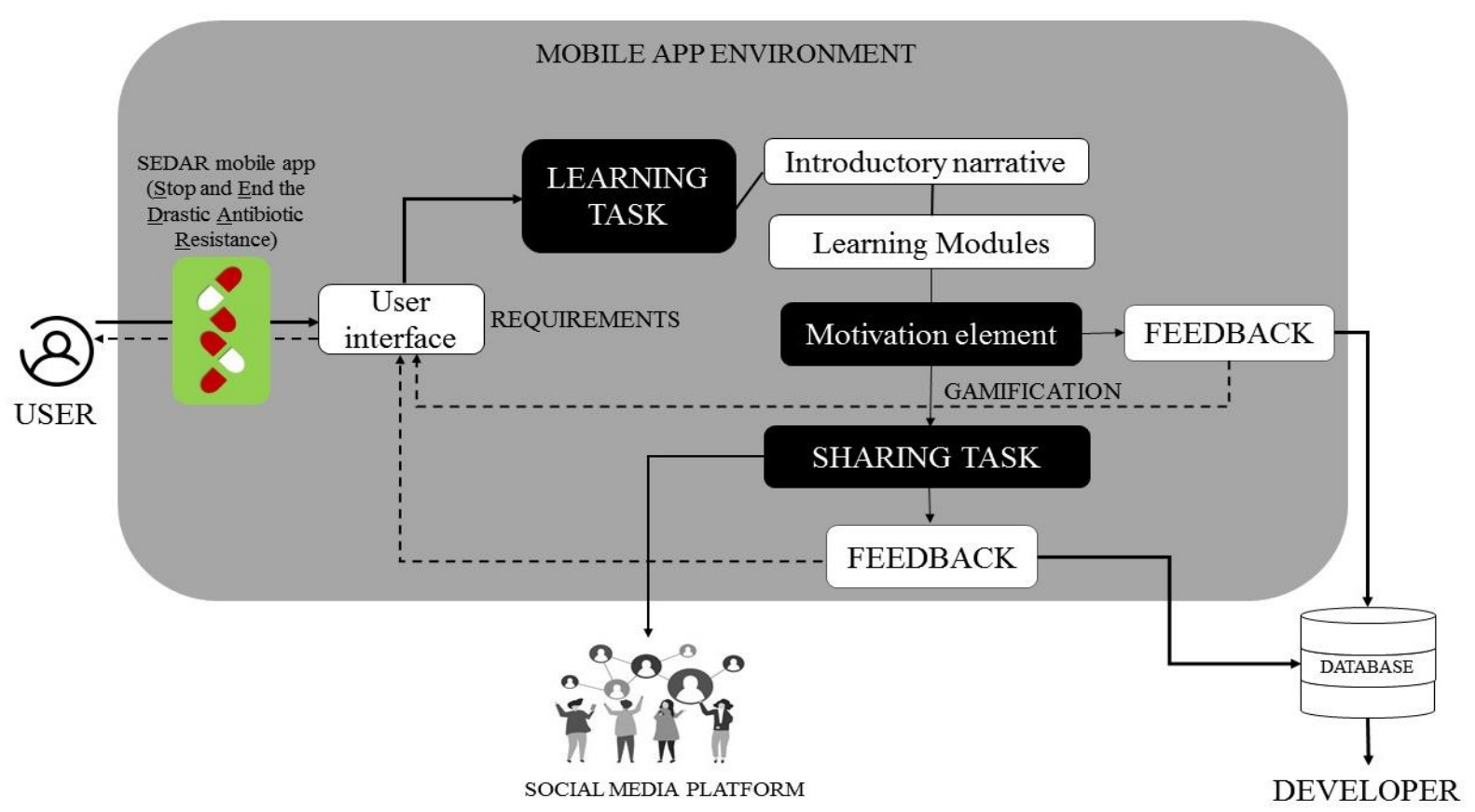

Figure 6: A Conceptual Model of an Application for Antibiotic Resistance Awareness

\section{Discussion}

Based on the requirement analysis, we identified two focal issues to be addressed through this awareness app; (i) the users' misunderstanding of using antibiotics to treat viral diseases and (ii) the users' tendency not to finish antibiotics. The first issue signified the need to educate the public that antibiotics are only effective in treating bacterial infections, and it is useless for viral diseases. Many users took antibiotics when they were suffering from common viral diseases such as fever, cold and sore throat. If this practice persisted, we might encounter more drastic occurrences of antibiotic-resistant bacteria. A similar concern is addressed in research by Hale et al. (2017) that evaluated the effectiveness of the 'Doctor Doctor' game to increase antibiotic awareness in children (Molnar, 2017).

The second issue highlighted the importance of finishing the antibiotics medication. This is because incomplete antibiotics consumption provides the opportunity for the remaining viable bacteria pathogens to strategize their way of combating antibiotics (Piddock, 2017). In the future, these antibiotics will no longer work against the bacteria, leaving us with difficulties to perform many medical procedures such as surgery and organ transplantations (Ventola, 2015). So far, there is no new class of antibiotics found for over 40 years (Buckland, 2017).

The requirement analysis indirectly depicted a low public awareness of antibiotics resistance issues in the community. This statement is justified because the level of education did not correlate with the awareness level. This again reflected that the existing awareness programs on antibiotic resistance were ineffective in disseminating antibiotics and antibiotics resistance information. However, this study is limited by the assumption that health practitioners were aware of the correct antibiotics handling practices. Since the antibiotics users were mostly dependent on their doctors to provide them with the needed treatment, there is still a concern 


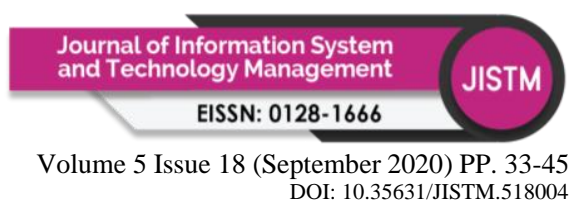

since antibiotics resistance can occur due to the incorrect practice by both parties; users or prescribers (Al-Juma et al., 2018; Salsgiver et al., 2018).

Since the target group for this application is the public, layman terms should be used instead of the scientific terms to improve the antibiotic resistance's understanding. The learning modules contain the antibiotic information, where each user will be given a choice to select the appropriate response based on the situation presented. At the same time, the gamification elements will provide feedback on the user's response. Sardi et al. (2017) highlighted that points, badges and leader boards were the most recurrent gamification elements used in many apps. For this conceptual model design, 'points' were chosen as the main gamification elements based on the following reasons; (i) points enable us to evaluate the awareness level based on the scores that measure the correct responses (Silpasuwanchai et al., 2016), (ii) points allow us to compare the progress (increased/decreased awareness level), measured from the previous scores and latest scores and (iii) points may serve as the starting point for more integration of gamification mechanics in the future such as leader boards.

\section{Conclusion}

In conclusion, the outcome from the requirement gatherings was sufficient to reveal the essential insights to design the antibiotic resistance awareness application's conceptual model. Since the participants' knowledge of antibiotics is often reflected in their attitude while using antibiotics, the model needs to embed two things in parallel, which are to educate for public awareness and encourage behavior changes. To support these needs, the conceptual model design incorporated gamification mechanics. This paper contributed to the design of a conceptual model that focuses on abstract health issues such as antibiotic resistance and may serve as the basis for developing other mHealth awareness applications.

\section{Acknowledgment}

This study is supported by Universiti Kebangsaan Malaysia under GGPM grant (GGPM-2017073).

\section{References}

Abu Hassan Shaari, N. S., Abdul Manaf, Z., Mohamad Ali, Nazlena Shahar, S., \& Mohamed Ismail, N. A. (2016). Usage of Mobile Applications in Diabetes Management. A Review Malaysian Journal of Health Sciences, 1-10.

Adams, A., \& Cox, A. L. (2008). Questionnaires, in-depth interviews and focus groups.

Afzal Khan, A. K., Banu, G., \& Reshma, K. K. (2013). Antibiotic resistance and usage-a survey on the knowledge, attitude, perceptions and practices among the medical students of a Southern Indian teaching hospital. Journal of Clinical and Diagnostic Research: JCDR, 7(8), 1613.

Al-Juma, M. A., Anderson, C., \& Boyd, M. J. (2018). Fever means antibiotic'the Omani public's attitudes to the use of antibiotics for treating the common cold-Selfcare Journal. SelfCare Journal.

Bodendorf, F., \& Kaiser, C. (2010). Detecting opinion leaders and trends in online communities. Proceedings of the 2010 Fourth International Conference on Digital Society, 124-129.

Boratto, L., Carta, S., Fenu, G., Manca, M., Mulas, F., \& Pilloni, P. (2017). The role of social interaction on users motivation to exercise: a persuasive web framework to enhance the self-management of a healthy lifestyle. Pervasive and Mobile Computing, 36, 98-114. 


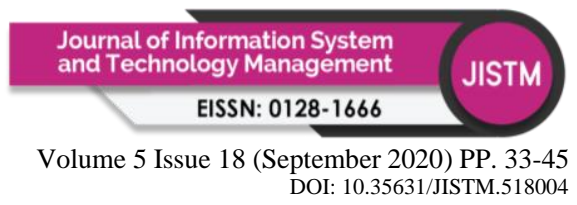

Brauner, P., Valdez, A. C., Schroeder, U., \& Ziefle, M. (2013). Increase physical fitness and create health awareness through exergames and gamification. In Human factors in computing and informatics (pp. 349-362). Springer.

Buckland, D. (2017). Antimicrobial resistance and the race to find new antibiotics. Prescriber, $28(1), 12-15$.

Carrino, S., Caon, M., Angelini, L., Mugellini, E., Khaled, O. A., Orte, S., Vargiu, E., Coulson, N., Serrano, J. C., \& Tabozzi, S. (2014). PEGASO: A personalized and motivational ICT system to empower adolescents towards healthy lifestyles. Innovation in Medicine and Healthcare.

Deterding, S., Dixon, D., Khaled, R., \& Nacke, L. (2011). From game design elements to gamefulness: defining gamification. Proceedings of the 15th International Academic MindTrek Conference: Envisioning Future Media Environments, 9-15.

Donnelly, L., Davies, M., \& Wasley, A. (2017). Soaring antibiotic resistance fuels steep rise in use of "last hope" drugs. http//www.telegraph.co.uk/science/2017/02/24/soaringantibiotic-resistance-fuels-steep-rise-use-last-hope/

Gooch, D., Vasalou, A., Benton, L., \& Khaled, R. (2016). Using gamification to motivate students with dyslexia. Proceedings of the 2016 CHI Conference on Human Factors in Computing Systems, 969-980.

Hale, A. R., Young, V. L., Grand, A., \& McNulty, C. A. M. (2017). Can Gaming Increase Antibiotic Awareness in Children? A Mixed-Methods Approach. JMIR Serious Games, $5(1)$.

Huang, Y., Gu, J., Zhang, M., Ren, Z., Yang, W., Chen, Y., Fu, Y., Chen, X., Cals, J. W. L., \& Zhang, F. (2013). Knowledge, attitude and practice of antibiotics: a questionnaire study among 2500 Chinese students. BMC Medical Education, 13(1), 163.

Islahudin, F., Madihah, A., Tamezi, A., \& Shah, N. M. (2014). Knowledge, Attitudes and Practices About Antibiotic Use Among the General Public in Malaysia. Southeast Asian Journal of Tropical Medicine and Public Health, 45(6), 1474.

Johnson, D., Deterding, S., Kuhn, K.-A., Staneva, A., Stoyanov, S., \& Hides, L. (2016). Gamification for health and wellbeing: A systematic review of the literature. Internet Interventions, 6, 89-106.

Jones, T. L., Baxter, M. A. J., \& Khanduja, V. (2013). A quick guide to survey research. The Annals of The Royal College of Surgeons of England, 95(1), 5-7.

Kao, H.-Y., Wei, C.-W., Yu, M.-C., Liang, T.-Y., Wu, W.-H., \& Wu, Y. J. (2018). Integrating a mobile health applications for self-management to enhance Telecare system. Telematics and Informatics, 35(4), 815-825.

Kim, H., Kang, J.-H., Park, H. A., Cho, S. H., Jeon, S., Jung, J., \& Sung, E. (2015). Development of a Smartphone Application Prototype for Child Obesity Prevention: Rationale and Study Design of Acceptability and Feasibility Tests. Korean Journal of Health Promotion, 15(4), 194-201.

Kim, S., \& Baek, T. H. (2018). Examining the antecedents and consequences of mobile app engagement. Telematics and Informatics, 35(1), 148-158.

Korn, O., \& Tietz, S. (2017). Strategies for Playful Design when Gamifying Rehabilitation: A Study on User Experience. Proceedings of the 10th International Conference on PErvasive Technologies Related to Assistive Environments, 209-214.

Lee, C., Lee, K., \& Lee, D. (2017). Mobile Healthcare Applications and Gamification for Sustained Health Maintenance. Sustainability, 9(5), 772.

Lim, K. K., \& Teh, C. C. (2012). A cross sectional study of public knowledge and attitude towards antibiotics in Putrajaya, Malaysia. Southern Med Review, 5(2), 26-33. 


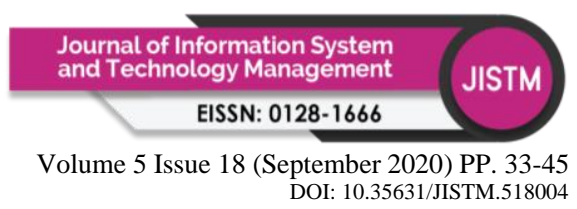

Man-Ching, Y., Shin, Y. C., \& Che Din, N. (2018). SpeakMore: A Mobile App towards Better Stuttering Awareness to Improve Speech Therapy. Proceedings of the 2018 International Conference on Health Informatics \& Medical Systems.

Molnar, A. (2017). Children as agents of change in combatting antibiotic resistance. Journal of Health Services Research \& Policy, 22(4), 258-260.

Morschheuser, B., Hamari, J., Werder, K., \& Abe, J. (2017). How to gamify? A method for designing gamification. Proceedings of the 50th Hawaii International Conference on System Sciences 2017.

O'Neill, J. (2016). Tackling drug-resistant infections globally: final report and recommendations. The Review on Antimicrobial Resistance.

Peng, W., Kanthawala, S., Yuan, S., \& Hussain, S. A. (2016). A qualitative study of user perceptions of mobile health apps. BMC Public Health, 16(1), 1158.

Piddock, L. J. V. (2017). Understanding drug resistance will improve the treatment of bacterial infections. Nature Reviews Microbiology, 15(11), 639.

Qamar, M., Sheikh Abdullah, N. H., Khan, J., Mahmud, A., \& Ahmad, A. (2014). Knowledge and Attitude Towards Antibiotic Usage Among General Public in Shah Alam, Malaysia. UK Journal of Pharmaceutical and Biosciences, 2(6), 60-66.

Richards, C., Thompson, C. W., \& Graham, N. (2014). Beyond designing for motivation: the importance of context in gamification. Proceedings of the First ACM SIGCHI Annual Symposium on Computer-Human Interaction in Play, 217-226.

Salsgiver, E., Bernstein, D., Simon, M. S., Eiras, D. P., Greendyke, W., Kubin, C. J., Mehta, M., Nelson, B., Loo, A., \& Ramos, L. G. (2018). Knowledge, Attitudes, and Practices Regarding Antimicrobial Use and Stewardship Among Prescribers at Acute-Care Hospitals. Infection Control \& Hospital Epidemiology, 39(3), 316-322.

Sardi, L., Idri, A., \& Fernández-Alemán, J. L. (2017). Gamified Mobile Blood Donation Applications. International Conference on Bioinformatics and Biomedical Engineering, 165-176.

Scaioli, G., Gualano, M. R., Gili, R., Masucci, S., Bert, F., \& Siliquini, R. (2015). Antibiotic use: a cross-sectional survey assessing the knowledge, attitudes and Practices amongst Students of a School of Medicine in Italy. PLoS One, 10(4), e0122476.

Serral, E., Valderas, P., \& Pelechano, V. (2010). Towards the model driven development of context-aware pervasive systems. Pervasive and Mobile Computing, 6(2), 254-280.

Silpasuwanchai, C., Ma, X., Shigemasu, H., \& Ren, X. (2016). Developing a comprehensive engagement framework of gamification for reflective learning. Proceedings of the 2016 ACM Conference on Designing Interactive Systems, 459-472.

Tarute, A., Nikou, S., \& Gatautis, R. (2017). Mobile application driven consumer engagement. Telematics and Informatics, 34(4), 145-156.

Thiruvanackan, K., \& Mohd. Yusof, M. (2017). Evaluation of mobile health (MHEALTH) application from the pharmacist perspective. Journal of Information System and Technology Management, 37-54.

Ventola, C. L. (2015). The antibiotic resistance crisis: part 1: causes and threats. Pharmacy and Therapeutics, 40(4), 277.

WHO. (2015). Antibiotic resistance: Multi-country public awareness survey.

WHO. (2016). Antibiotic resistance. In World Health Organisation.

Zulkifli, M. N., Mohd Yusof, U. N., Zakaria, Munirah Rosli, Nurshuhadah z Mohd Abdul Azis, M. M., Nasarudin, Rona Asnida Sheikh Abdullah, S. N. H., Bedir, Y., Kurniawan, R., W Zaki, M. D., Hussain, A., \& Che Hamzah, J. (2018). Take your meds!: a prototype medication reminder mobile phone application. 10th Medical Undergraduates' Annual Scientific Research Meeting. 